\title{
Research on Chinese Medicine Honeysuckle Medicinal Ingredients and Pharmacological Effects
}

\author{
Yumei Wang \\ Medical College, Hexi University, 24 Danxia East Road, Zhangye 734000, PR China
}

\begin{abstract}
Honeysuckle is a medicinal plant of Chinese medicine, sweet, cold, into the heart, lung, stomach, the main effect of heat detoxification, anti-inflammatory and anti-bacterial, hemostasis, anti-virus, fever, fever, cancer and other diseases Have a good application effect. The honeysuckle is sweet and cold, it contain flavonoids, volatile oil, organic acids and inorganic compounds such as inorganic compounds and other active ingredients, than blood, detoxification, gallbladder, liver and anti-virus and other effects, honeysuckle in a variety of traditional Chinese medicine preparations to be applied. With the deepening of research, honeysuckle will be in the antibacterial and antiviral clinical treatment shows a more obvious medicinal value. Chinese medicine honeysuckle contains flavonoids, organic acids, volatile oil, etc. the clinical pharmacological effects are generally detoxification, anti-inflammatory, anti-lipid, anti-endotoxin, antioxidant and so on. Chinese herbal medicine honeysuckle contain very large Chinese and Western medicine compound preparation value, it has a very important clinical value, which can be vigorously promoted with clinical significance.
\end{abstract}

Keywords: Honeysuckle, Medicinal Ingredients, Pharmacological Effects

\section{Theoretical Introduction of Honeysuckle}

Honeysuckle also known as the second treasure, silver flower, it is a common clinical Chinese medicine plants, the main effect is detoxification, blood, sparse heat, etc., with a national treasure a flower of the name. Honeysuckle because of a variety of medicinal ingredients and pharmacological effects, many of the current clinical drug preparations contain honeysuckle ingredients, honeysuckle has become Chinese key management of valuable medicines. This article mainly discusses the medicinal composition and clinical pharmacological effect of honeysuckle.

Honeysuckle flowers and rattan have a certain effect, especially the strongest effect of flowers. In clinical pharmaceutical preparations, the vast majority of preparations contain honeysuckle ingredients. As the medicinal ingredients of honeysuckle more pharmacological effects, so honeysuckle has been frequent applications, and become a national key management, the State Council clearly defined one of the valuable medicinal herbs ${ }^{[1]}$.

In order to further explore the medicinal ingredients and clinical pharmacology contained in traditional Chinese medicine honeysuckle, the literature and medical materials of honeysuckle in recent years have been studied. Through the pharmacological study of Chinese herbal medicine honeysuckle, quality and chemical composition research, vegetation research, statistics, summed up the medicinal ingredients of honeysuckle, clinical pharmacological effects. Honeysuckle cold, sweet, into the lungs, heart, stomach, with detoxification, anti-inflammatory, tonic treatment of wind effect, Indications full of disease, fever, fever, carbuncle and tumor embolism. For the dizziness, dry mouth, thirsty, sweating and boring, enteritis, bacillary dysentery, measles, pneumonia, tonsillitis and other diseases have a certain effect. On the whole, the flavonoids in honeysuckle have antipyretic and antiinflammatory effects; the volatile oil has the hemostatic effect; the triterpenoid has antiviral effect; the inorganic substance has the effect of defoaming the liver and the organic acid has the antibacterial effect.

\section{Analysis of Honeysuckle Medicinal Ingredients}

A large number of studies have shown that honeysuckle contains protocatechuic acid, chlorogenic 
acid, chlorogenic acid and other organic acids compounds, of which the highest content of chlorogenic acid. Chlorogenic acid has the effect of lowering blood pressure, lowering blood fat, anti-tumor, anti-oxidation, anti-virus and antibacterial effect, its antioxidant effect than tocopherol, ferulic acid, acid and other substances are also strong. Studies have shown that chlorogenic acid has a strong antioxidant effect because the R-0 H-containing radicals can promote the formation of hydrogen free radicals, reduce the activity of oxygen free radicals, and thus play a role in the antioxidant effect. In addition, chlorogenic acid also has the effect of inhibiting hyaluronidase activity. Protocatechuic acid can inhibit E. coli, Proteus, Pseudomonas aeruginosa breeding, its small only has the role of improving cardiac function, but also treatment of patients due to hypoxia caused by abnormal blood pressure ${ }^{[2]}$.

Studies have pointed out that from the honeysuckle can be extracted from benzoic acid vinegar, phenylethyl alcohol, linolenic acid methyl ester and linolenic acid vinegar and other volatile oil. These volatile oils are mainly extracted from honeysuckle flowers, branches and vines. As the honeysuckle volatile oil has a strong lipophilic, and the boiling point is low, it can be steam distillation, carbon dioxide extraction and other methods to extract. Technical staff in the extraction of honeysuckle volatile oil should control the heating temperature, if the temperature is too high, it will greatly reduce the honeysuckle oil rate.

Honeysuckle contains peach, luteolin and other flavonoids. Among them, luteolin small only inhibit the virus and the role of bacterial reproduction (such as inhibition of poliovirus, coli and simple herpes simplex virus), but also to prevent liver fibrosis. Hypericum has analgesic, anti-inflammatory, antispasmodic and diuretic effect, the analgesic effect is better than aspirin. Honeysuckle has the role of protecting the liver, reducing liver damage and promoting bile secretion. The methods for extracting triterpenoids from honeysuckle are distillation, steam distillation, freezing and extraction. Related studies have shown that triterpenoids have expectoration, than cough, drive, sweat, deworming and analgesic effects. Honeysuckle contains inorganic components such as flavonoids, triterpenoids, volatile oils and organic acids, and contains inorganic components such as $\mathrm{Ni}, \mathrm{PU}, \mathrm{Mn}, \mathrm{Li}, \mathrm{Co}, \mathrm{Sr}, \mathrm{Cr}, \mathrm{Ca}, \mathrm{V}, \mathrm{Ba}, \mathrm{Cu}$ and other inorganic elemental compounds ${ }^{[3]}$.

\section{Analysis of Honeysuckle Pharmacological Effects}

Studies have shown that honeysuckle contains chlorogenic acid components can inhibit Sarkozy virus and respiratory syncytial virus breeding, commonly used honeysuckle preparations to treat respiratory diseases. There are scholars in the process of treatment of patients with respiratory tract infection, the use of injection for treatment of patients set as the treatment group, the use of penicillin for treatment of patients as a control group. Honeysuckle can produce a strong inhibitory effect on CC14, thereby effectively reducing the CC14 liver damage, with the role of liver protection. In addition, chlorogenic acid also has the role of gallbladder. Some scholars in the course of treatment of patients with biliary, the use of gall bladder soup for treatment of patients set as the treatment group, the use of gallstone tongli tablets for treatment of patients as a control group. The results of treatment showed that the total effective rate of treatment in patients treated with treatment was significantly higher than that in the control group, indicating that lonicum decoction containing honeysuckle had a strong biliary effect ${ }^{[4]}$.

Honeysuckle preparations can inhibit cholera, staphylococcus, pneumonia, Salmonella typhi and other bacteria breeding, and can effectively resist Mycobacterium tuberculosis and Pseudomonas aeruginosa and other bacteria attack. Some scholars in the treatment of rotavirus enteritis patients in the process, the use of Shuanghuanglian powder injection treatment of patients set to honeysuckle group, the use of conventional drugs for treatment of patients as a control group. The results of treatment showed that the total effective rate of honeysuckle group was significantly higher than that of the control group, which indicated that the double-yellow powder with honeysuckle composition had strong antibacterial effect. Fig.1 shows the clinical pharmacological effects. 


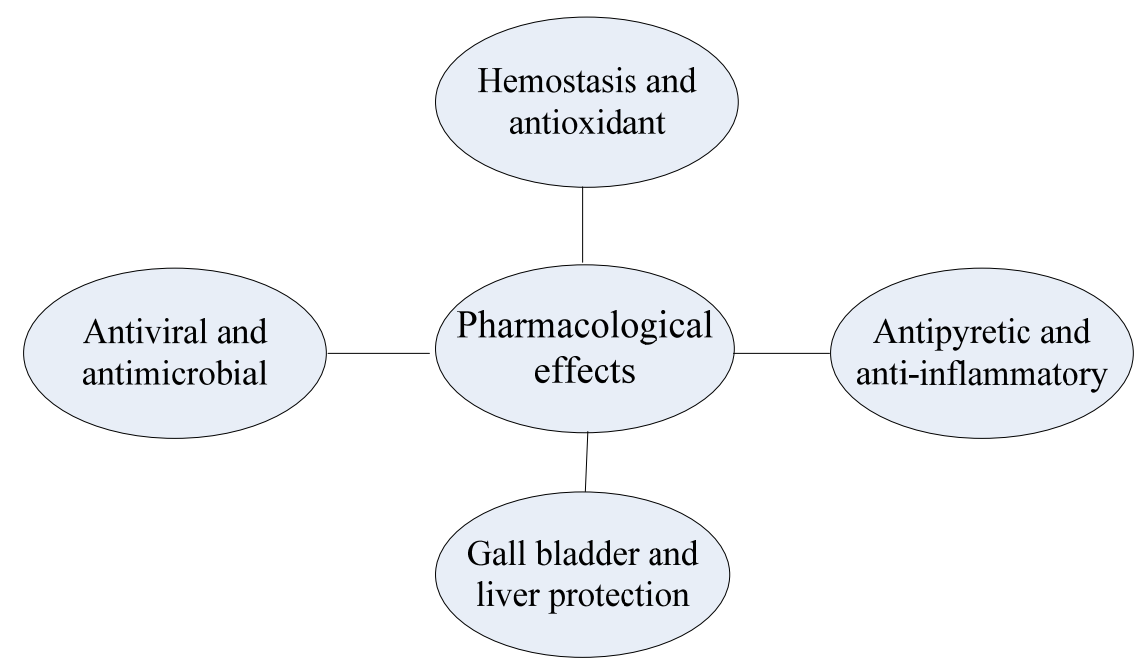

Fig. 1.The clinical pharmacological effects

Honeysuckle preparations in the anti-inflammatory effect and dermatitis flat, dexamethasone and other drugs similar. Relevant reports pointed out that the use of honeysuckle capsules for patients with chronic pharyngitis after treatment, the total effective rate of $90 \%$, which shows that honeysuckle ingredients containing honeysuckle capsules with antipyretic effect. In addition, the relevant reports indicate that honeysuckle also has more than blood efficacy. Hemostatic effect: the use of honeysuckle for charcoal decoction has obvious hemostatic effect, the reason is that honeysuckle contains caffeic acid, chlorogenic acid medicinal ingredients. Chlorogenic acid and caffeic acid is the active ingredient of honeysuckle hemostatic effect, which is helpful to shorten the time of coagulation and bleeding time. Antioxidant analysis: honeysuckle has antioxidant effect, because it contains flavonoids. Honeysuckle water extract can directly remove hydrogen peroxide, to a certain extent, reduce the scald in mice to release lysin and the ability to synthesize neutrophils, fully confirmed the honeysuckle antioxidant effect.

\section{Product Quality Analysis}

Honeysuckle contains chlorogenic acid, 1.5\%, pharmacopoeia at the same time on the content of active ingredients and specific changes in the provisions of the provisions for the evaluation of the quality of the drug provides a method. The quality of honeysuckle was affected by many factors. The main factors were as follows: the quality was affected by the origin and the source of the plant, the different origin, the different origin and the honeysuckle were different, and the honeysuckle had more influence than the variety ${ }^{[5]}$.

The results showed that the content of chlorogenic acid decreased with the blooming period, and the oil consumption increased gradually from the honeysuckle buds to the blooming stage, which was affected by the harvesting period, the growth environment, the development stage and the storage time. According to the order of flowers, two white, white and three blue buds picking is to improve the quality and yield the best time. Growth environment also honeysuckle flower quality has an important impact, and sunflower flower chlorogenic acid content was significantly higher than the shady slope products. Honeysuckle in the day the chlorogenic acid content is also different, of which 11:00 - 13:00 is the best harvest time; harvesting and processing methods on the honeysuckle quality also has a significant impact, different phenological picking honeysuckle quality and The yield and quality of the first stage were the highest, and the yield and quality of the flower decreased obviously with the increase of the number of picking.

The results of different processing methods found that smoked products and non-smoked products are not chlorogenic acid and chlorogenic acid no significant difference, and smoked after the flowers more vivid colors; steam drying product quality was better than the students drying products, compared with the sun smelting chlorogenic acid and total volatile oil content are higher than other centralized production methods, while improving the production of the product sense of concern. The quality of herbs was affected by the method of extraction and determination. The 
quality of honeysuckle was higher than that of raw honeysuckle. Some studies have shown that water extraction and alcohol extraction method is better. Chemical composition research, honeysuckle mainly mushrooms, flavonoids, volatile oil, organic acids and inorganic elements. In this paper, the honeysuckle extraction and separation technology has appeared in the capillary zone electrophoresis method, which provides better application value for quality control ${ }^{[6]}$.

\section{Conclusions}

Honeysuckle is a medicinal plant, with sweet, cold and other characteristics, in anti-inflammatory, detoxification, anti-bacterial and liver and gallbladder, etc. are very strong effect, a national treasure a flower, medicine shop small gods and other reputation. In clinical, honeysuckle can be commonly used on the tumor, fever and respiratory infections and other diseases for treatment. Commonly used honeysuckle preparations are silver yellow injection, Shuang huang lian injection, silver yellow film, Victoria C Yin qiao tablets, silver yellow and other films. In recent years, with the continuous development of China's pharmacy, medical workers should be small cut honeysuckle potential medicinal value, give full play to its anti-virus, antipyretic, anti-inflammatory and other pharmacological effects in modern Chinese and Western medicine preparations, the vast majority of preparations contains honeysuckle, such as Shuang huang lian injection and so on. With the modern pharmacy in-depth study, honeysuckle in anti-inflammatory, anti-bacterial and anti-drug and other areas of medicinal value will be greater.

\section{References}

[1] Yang Peng. Chinese medicine honeysuckle medicinal ingredients and pharmacological effects[J]. Chinese community physician, 2013, 15 (15): 24.

[2] Jing Hengcui, Zhai Qingmei, Cao Qian. Chinese Medicinal Ingredients and Pharmacological Analysis of Honeysuckle [J]. Henan Traditional Chinese Medicine, 2014, 3 (7): 63-64.

[3] Wang Yadan. Yang Jianbo. Dai Zhong. Etal. Chinese medicine honeysuckle research progress [J]. Chinese Journal of Drug Analysis .2014 (11); 1928-1935.

[4] Jing Hengcui, Zhai Qingmei, Cao Qian. Chinese medicinal ingredients and pharmacological analysis of honeysuckle [J]. Henan Traditional Chinese Medicine, 2014, 09 (12) 1839-1840.

[5] CHEN Ji-ming, HONG Chao qun. Study on pharmacological effects of honeysuckle [J]. Asia Pacific Traditional Medicine, 201 5, 6 (2): 4 5- 46.

[6] JIANG Nan-hui. Studies on chemical constituents of honeysuckle [J]. Chinese Traditional Medicine, 2015, 3(2): 22-23. 